\title{
TOPOLOGICAL ALGEBRAS WTTH A GIVEN DUAL
}

\author{
AJIT KAUR CHILANA ${ }^{1}$
}

\begin{abstract}
Given an algebra $E$ and a total subspace $E^{\prime}$ of its algebraic dual, we obtain necessary and sufficient conditions in terms of $E^{\prime}$ for the existence of an $A$-convex or a locally $m$-convex topology on $E$ compatible with duality $\left(E, E^{\prime}\right)$. It has also been proved that if $E$ with the weak topology $w\left(E, E^{\prime}\right)$ is the closed linear hull of a bounded set and has hypocontinuous multiplication then it is locally $m$-convex.
\end{abstract}

1. Introduction. Let $E$ be a complex (or real) algebra and $E^{\prime}$ be a total subspace of the algebraic dual $E^{*}$. To avoid repetitions we use the notation, terminology and results in [3] and [4] without specifications. An algebra with a locally convex linear topology for which multiplication is separately continuous will be called a locally convex algebra. An absolutely convex set $B$ in $E$ is called right (left) $A$-convex if it absorbs $B x(x B)$ for each $x \in E$, it will be called $A$-convex if it is both right and left $A$ convex. A locally convex algebra is called (right, left) A-convex if there exists a basis of (right, left) $A$-convex neighbourhoods of zero. Multiplication in a locally convex algebra will be said to be right (left) hypocontinuous if given a neighbourhood $U$ of $o$ and a bounded set $B$ there exists a neighbourhood $V$ of $o$ satisfying $V B \subset U(B V \subset U)$. We say that multiplication is hypocontinuous if it is both right and left hypocontinuous. Gulick [5] has, however, called right hypocontinuity by hypocontinuity.

In $\$ 2$ we answer the following question asked by Cochran [4].

(3.7) Under what conditions, in terms of $E^{\prime}$, does $\Sigma\left(E, E^{\prime}\right)$ or $\chi\left(E, E^{\prime}\right)$ the finest $A$-convex or locally $m$-convex topology on $E$ compatible with duality $\left(E, E^{\prime}\right)$-exist?

It is known ([3] and [9], MR 41 \#7435) that for $E$ with the weak topology $w\left(E, E^{\prime}\right)$ the conditions of joint continuity of multiplication, of $A$ convexity and of local $m$-convexity are mutually equivalent. We prove

Received by the editors December 14, 1972 and, in revised form, March 19, 1973. AMS (MOS) subject classifications (1970). Primary 46H05.

$K e y$ words and phrases. (right, left) $A$-convex algebra, locally $m$-convex algebra, topology compatible with duality, (right, left) multiplicative translate of a functional, collectionwise multiplicative set of functionals, collectionwise (right, left) multiplicativetranslation invariant set of functionals, hypocontinuous and jointly continuous multiplication.

${ }^{1}$ Research supported in part by UGC Grant F-U-2/71 (Sch). 
in $\S 3$ that if $\left(E, w\left(E, E^{\prime}\right)\right)$ is the closed linear hull of a bounded subset of itself then the condition of hypocontinuity of multiplication is also equivalent to all these conditions.

For $y \in E$ and $f \in E^{*}$, the right $y$-multiplicative translate $f_{y}$ and the left $y$-multiplicative translate ${ }_{y} f$ of $f$ are given by $f_{y}(x)=f(x y)$ and ${ }_{y} f(x)=f(y x)$ for $x \in E$ respectively. For $y \in E$ and $S \subset E^{*}$, let $S(y)=\{f(y): f \in S\}$, $S_{y}=\left\{f_{y}: f \in S\right\}$ and ${ }_{y} S=\left\{{ }_{y} f: f \in S\right\}$.

2. Topologies on $E$ compatible with duality $\left(E, E^{\prime}\right)$.

(2.1) DEFINITION. A set $S \subset E^{*}$ is called collectionwise multiplicative if $S(x y) \subset S(x) S(y)$ for all $x, y \in E$.

(2.2) Definition. A set $S \subset E^{*}$ is called collectionwise right (left) multiplicative-translation invariant if for each $y \in E$ there is $\rho_{y} \geqq 0$ satisfying $\left.S_{y}(x) \subset \rho_{y} S(x){ }_{y} S(x) \subset \rho_{y} S(x)\right)$ for all $x \in E$. $S$ will be called collectionwise multiplicative-translation invariant if it is both collectionwise right and collectionwise left multiplicative-translation invariant.

It is easy to see that every collection of multiplicative linear functionals is collectionwise multiplicative and every balanced, $w\left(E^{*}, E\right)$-bounded, collectionwise multiplicative subset of $E^{*}$ is collectionwise multiplicativetranslation invariant. Also an arbitrary union of collectionwise multiplicative sets is collectionwise multiplicative and a finite union of balanced collectionwise (right, left) multiplicative-translation invariant sets is collectionwise (right, left) multiplicative-translation invariant.

(2.3) Lemma. Let $S \subset E^{\prime}$ be balanced and $w\left(E^{\prime}, E\right)$-compact, and let $S^{\circ}$ be its polar in $E$.

(i) $S^{\circ}$ is idempotent if and only if $S$ is collectionwise multiplicative.

(ii) $S^{\circ}$ is (right, left) A-convex if and only if $S$ is collectionwise (right, left) multiplicative-translation invariant.

Proof. (i) Sufficiency is clear.

Necessity. For $x \in E$, let $p(x)=\sup \{|f(x)|: f \in S\}$. Since $S$ is $w\left(E^{\prime}, E\right)$ compact, $p(x)<\infty$ and there is an $f \in S$ (depending on $x$ ) satisfying $p(x)=|f(x)|$. Because $S$ is balanced, $g=$ signum $f(x) \cdot f$ is in $S$. So $p(x)=$ $g(x)$ for some $g$ in $S$. Also $S^{\circ}=\{x \in E: p(x) \leqq 1\}$ and $p$ is its Minkowski functional. Now $S^{\circ}$ is idempotent, so $p$ is submultiplicative i.e. $p(x y) \leqq$ $p(x) p(y)$ for all $x, y$ in $E$.

Let $x, y \in E$ and $f \in S$. Then $|f(x y)| \leqq p(x) p(y)$. So there is a scalar $\lambda$ such that $|\lambda| \leqq 1$ and $f(x y)=\lambda p(x) p(y)$. Also there exist $g$ and $h$ in $S$ (depending on $x$ and $y$ respectively) satisfying $p(x)=g(x)$ and $p(y)=h(y)$. If $g_{1}=\lambda g$ then $g_{1} \in S$. Thus $f(x y)=g_{1}(x) h(y) \in S(x) S(y)$. Hence $S(x y) \subset$ $S(x) S(y)$ for all $x, y \in E$ and $S$ is collectionwise multiplicative.

(ii) Sufficiency is clear. 
Suppose $S^{\circ}$ is right $A$-convex. For $y \in E$ there is $\lambda_{y}>0$ such that $S^{\circ} y \subset \lambda_{y} S^{\circ}$. If $p$ is as in the proof of (i) above then $p$ satisfies all other properties except that submultiplicativity is replaced by $p(x y) \leqq \lambda_{y} p(x)$ for all $x, y \in E$. So $|f(x y)| \leqq p(x y) \leqq \lambda_{y} p(x)$. Therefore, $f(x y)=\mu \lambda_{y} p(x)$ for some $\mu$ with $|\mu| \leqq 1$. Let $g_{2}=\mu g$, where $g \in S$ is such that $p(x)=g(x)$. Then $f(x y)=\lambda_{y} g_{2}(x)$. So $S(x y) \subset \lambda_{y} S(x)$ for all $x, y \in E$. Hence $S$ is collectionwise right multiplicative-translation invariant. Similarly we can prove for other parts.

(2.4) THEOREM. There exists a locally m-convex topology on $E$ compatible with duality $\left(E, E^{\prime}\right)$ if and only if there exists a family $\mathscr{S}$ of absolutely convex, $w\left(E^{\prime}, E\right)$-compact, collectionwise multiplicative sets in $E^{\prime}$ that cover $E^{\prime}$.

(2.5) Corollary. The Mackey topology $\tau\left(E, E^{\prime}\right)=\chi\left(E, E^{\prime}\right)$ if and only if every absolutely convex, $w\left(E^{\prime}, E\right)$-compact set is contained in some absolutely convex, $w\left(E^{\prime}, E\right)$-compact, collectionwise multiplicative set in $E^{\prime}$.

(2.6) ThEOREM. There exists a (right, left) A-convex topology on $E$ compatible with duality $\left(E, E^{\prime}\right)$ if and only if there is a family $\mathscr{S}$ of absolutely convex, $w\left(E^{\prime}, E\right)$-compact, collectionwise (right, left) multiplicativetranslation invariant sets in $E^{\prime}$ that cover $E^{\prime}$.

(2.7) CoROllaRY. $\tau\left(E, E^{\prime}\right)=\Sigma\left(E, E^{\prime}\right)$ if and only if every absolutely convex, $w\left(E^{\prime}, E\right)$-compact subset of $E^{\prime}$ is contained in some absolutely convex, $w\left(E^{\prime}, E\right)$-compact, collectionwise multiplicative-translation invariant set.

(2.8) Remark. Since the existence of $\chi\left(E, E^{\prime}\right)\left(\Sigma\left(E, E^{\prime}\right)\right)$ is equivalent to the existence of some locally $m$-convex ( $A$-convex) topology on $E$ compatible with $\left(E, E^{\prime}\right)$, Theorems (2.4) and (2.6) give an answer to question (3.7) in [4].

(2.9) REMARK. If there are both $A$-convex and locally $m$-convex topologies on $E$ compatible with $\left(E, E^{\prime}\right)$ then $\chi\left(E, E^{\prime}\right)=\Sigma\left(E, E^{\prime}\right)$ if and only if every absolutely convex, $w\left(E^{\prime}, E\right)$-compact, collectionwise multiplicative-translation invariant set in $E^{\prime}$ is contained in an absolutely convex, $w\left(E^{\prime}, E\right)$-compact, collectionwise multiplicative set in $E^{\prime}$. This gives a partial answer to problem (3.6) in [4].

(2.10) ExAmple. Let $E$ be the algebra of complex (or real) polynomials without constant term and $E^{\prime}$ be the subspace of $E^{*}$ generated by $\left\{g_{i}\right.$ : $i=1,2, \cdots\}$, where $g_{i}\left(e_{j}\right)=\delta_{i j}, \quad e_{j}(x)=x^{j}$ for $i, j=1,2, \cdots$. Then $\left(E, w\left(E, E^{\prime}\right)\right)$ is a locally $m$-convex algebra having no nonzero continuous multiplicative linear functionals (see Proposition 3 and discussion thereafter in [8]). By Theorem (2.4) there is a family $\mathscr{S}$ of absolutely convex, 
$w\left(E^{\prime}, E\right)$-compact, collectionwise multiplicative sets in $E^{\prime}$ that cover $E^{\prime}$. In fact, if $G_{n}=\left\{n g_{i}: 1 \leqq i \leqq n\right\}$, then its absolutely convex, $w\left(E^{\prime}, E\right)$ closed hull $H_{n}$ in $E^{\prime}$ is $w\left(E^{\prime}, E\right)$-compact. Also the polar $G_{n}^{\circ}$ of $G_{n}$ in $E$ is idempotent and $H_{n}^{\circ}=G_{n}^{\circ}$. So by Lemma (2.3), $H_{n}$ is collectionwise multiplicative.

This example shows that a collectionwise multiplicative set need not contain even a single nonzero multiplicative linear functional.

(2.11) ExAmple. Let $E$ be the algebra $m$ of bounded complex (or real) sequences with pointwise addition and multiplication and let $E^{\prime}$ be the space $l_{1}$ of absolutely summable sequences. Then the Mackey topology $\tau\left(E, E^{\prime}\right)$ is the same as the strict topology $\beta$ on $E$ considered as the space $C_{b}(S)$ of bounded continuous complex (or real) functions on the space $S$ of positive integers with the discrete topology ([2], [3], and [4]). Let $\kappa$ be the compact open topology on $E$. By Corollary (3.3) in [4], there is no locally $m$-convex topology on $E$ between $\beta$ and $\kappa$. The dual of $(E, \kappa)$ is the space of sequences with only a finite number of nonzero elements and therefore $\kappa<w\left(E, E^{\prime}\right)$.

(i) $E$ is not locally $m$-convex under any topology compatible with $\left(E, E^{\prime}\right)$. So there exists no family of absolutely convex, $w\left(l_{1}, m\right)$-compact (and therefore, $\|\cdot\|_{1}$-compact), collectionwise multiplicative sets that cover $l_{1}$.

(ii) $(E, \beta)$ has the Mackey topology and is $A$-convex [4]. So every absolutely convex, $w\left(l_{1}, m\right)$-compact subset of $l_{1}$ is contained in an absolutely convex, $w\left(l_{1}, \boldsymbol{m}\right)$-compact, collectionwise multiplicative-translation invariant set.

3. $E$ with the weak topology $w\left(E, E^{\prime}\right)$. In this section $E$ will denote the space $E$ with the weak topology $w\left(E, E^{\prime}\right)$. For $B \subset E$ let $E_{B}$ denote the linear hull of $B$.

(3.1) Lemma. Suppose that $E$ has hypocontinuous multiplication. Let $g$ be in $E^{\prime}$ and $B$ be an absolutely convex bounded subset of $E$. Then the kernel $K(g)$ of $g$ contains a closed subspace $J$ of finite codimension in $E$ such that $K(g)$ contains $J E_{B}$ and $E_{B} J$.

Proof. Let $V$ be the polar of $\{g\}$ in $E$. Since the multiplication in $E$ is hypocontinuous there exists a finite set $F=\left\{f_{i}: 1 \leqq i \leqq n\right\}$ such that $V \supset\left(B F^{\circ}\right) \cup\left(F^{\circ} B\right)$. Let $J=\left\{x \in E: f_{i}(x)=0,1 \leqq i \leqq n\right\}$. Then $J B \subset F^{\circ} B \subset V$ and also $J$ is a closed subspace of finite codimension in $E$. Also $J E_{B}=J B \subset$ $V=\{g\}^{\circ}$ and as $J E_{B}$ is a linear space $J E_{B} \subset K(g)$. Similarly $E_{B} J \subset K(g)$.

(3.2) THEOREM. If $E$ is the closed linear hull of a bounded subset of itself and $E$ has hypocontinuous multiplication then $E$ has jointly continuous multiplication. 
Proof. Let $B$ be an absolutely convex bounded subset of $E$ such that $E=E_{B}^{-}$, where "-, denotes the closure in $E$. Let $g$ be in $E^{\prime}$. Let $J$ be as in

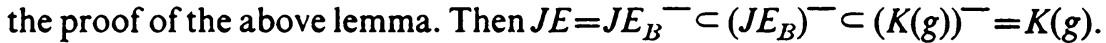
Similarly, $E J \subset K(\mathrm{~g})$. Theorem 2 of Warner [8] now gives that $E$ has jointly continuous multiplication.

(3.3) COROllary. If $E$ is the closed linear hull of a bounded set then $E$ is locally $m$-convex if and only if $E$ is $A$-convex if and only if it has jointly continuous multiplication if and only if it has hypocontinuous multiplication.

Proof. Combine Theorem (3.4) in [3], Theorem 1 in [9] and Theorem (3.2) above.

(3.4) REMARK. If a locally convex Hausdorff space is the closed linear hull of a bounded set i.e. it is boundedly generated (in short, BG) in the terminology of [6] then it is BG under each topology compatible with duality (Remark 10 in [1]). Every normed linear space is $B G$ and a product of BG spaces is again BG [6] (see also Remark 10 in [1] and [2]). Thus our results are applicable to a large class of algebras.

(3.5) ExAmple. The algebra $\left(\boldsymbol{m}, \boldsymbol{w}\left(\boldsymbol{m}, l_{1}\right)\right)$ is BG but not locally $m$-convex ([2], and Example (2.11) (i) above). So it is not $A$-convex and does not have hypocontinuous multiplication.

(3.6) ExAmpLe. Let $E$ be the algebra of all complex (or real) continuous functions on the interval $[0,1]$ with pointwise addition and multiplication equipped with the weak topology resulting from the sup norm topology. Then $E$ is a BG space. Warner [8] has shown that $E$ does not have jointly continuous multiplication. Therefore, $E$ is not $A$-convex and $E$ does not have hypocontinuous multiplication. Thus the claim made in the second part of Examples 3.12 in [5] is not valid.

(3.7) ExAmple. Consider the algebra $\varphi$ of complex (or real) sequences with only a finite number of nonzero elements. Then its algebraic dual is the space $\omega$ of all complex (or real) sequences under the duality given by $f(x)=\sum_{n=1}^{\infty} \xi_{n} \zeta_{n}$ for $x=\left(\xi_{n}\right) \in \varphi$ and $f=\left(\zeta_{n}\right) \in \omega$. So the Mackey topology $\tau(\varphi, \omega)$ is the finest locally convex topology on $\varphi$ and therefore is the same as the direct sum topology. Also bounded sets are finitedimensional and every absolutely convex absorbent set is a neighborhood of $o$ in $\varphi$. Moreover, $\omega$ is the $\alpha$-dual of $\varphi$ and $\tau(\varphi, \omega)$ is the same as the normal topology, a base of neighbourhoods of $o$ which is given by

$$
\left\{U_{f}=\left\{x=\left(\xi_{n}\right) \in \varphi: \sum_{n=1}^{\infty}\left|\xi_{n} \zeta_{n}\right| \leqq 1\right\}, f=\left(\zeta_{n}\right) \in \omega\right\} \quad[7, \S 30.1] .
$$

Let $V_{f}=\left\{x \in \varphi: \sum_{n=1}^{\infty}\left|\xi_{n} \zeta_{n}\right| \leqq 1, \sum_{n=1}^{\infty}\left|\xi_{n} \eta_{n} \zeta_{n}\right| \leqq \sum_{n=1}^{\infty}\left|\eta_{n} \zeta_{n}\right|\right.$ for all $y=$ $\left.\left(\eta_{n}\right) \in \varphi\right\}$. Then $V_{f} V_{f} \subset V_{f} \subset U_{f}$ and also $V_{f}$ is an absolutely convex 
absorbent set and thus a neighbourhood of $o$ in $\tau(\varphi, \omega)$. So $\tau(\varphi, \omega)$ is locally $m$-convex.

Now let $E$ denote the space $\varphi$ with the weak topology $w(\varphi, \omega)$. Then $E$ has hypocontinuous multiplication but does not have jointly continuous multiplication.

If $B$ is bounded on $E$ then there exists an integer $N$ and an $\alpha \geqq 0$ such that $B \subset\left\{x=\left(\xi_{n}\right): \xi_{n}=0\right.$ for $n>N$ and $\left|\xi_{n}\right| \leqq \alpha$ for $\left.n \leqq N\right\}$. Let $f=\left(\zeta_{n}\right) \in E^{\prime}=\omega$ and let $U$ be its polar in $E$. For $n \leqq N$, let $g_{n} \in E^{\prime}$ be given by $g_{n}(x)=$ $N \alpha\left|\zeta_{n}\right| \xi_{n}, x=\left(\xi_{n}\right) \in E$. Then the polar $V$ of $\left\{g_{n}: 1 \leqq n \leqq N\right\}$ is a neighbourhood of $o$ in $E$. Also $V B \subset U$. Thus $E$ has hypocontinuous multiplication.

Now consider $f=\left(\zeta_{n}\right) \in E^{\prime}$ given by $\zeta_{n}=1$ for all $n$. If $E$ is locally $m$-convex then by Theorem 1 of [8], the kernel $K(f)$ of $f$ contains an ideal $J$ of finite codimension. Let $x(\neq 0) \in J$. Let $y=\left(\eta_{n}\right) \in E$ be given by $\eta_{n}=\bar{\xi}_{n}$ $(n=1,2, \cdots)$. Then $x y \in J$. Now $f(x y)=\sum_{n=1}^{\infty}\left|\xi_{n}\right|^{2} \neq 0$. So $x y \notin K(f)$, which gives a contradiction. So $E$ is not locally $m$-convex and is, therefore, not $A$-convex and does not have jointly continuous multiplication.

I should like to thank the referee for useful comments and suggestions.

\section{REFERENCES}

1. A. K. Chilana, Invariant subspaces for linear operators in locally convex spaces, J. London Math. Soc. (2) 2 (1970), 493-503.

2. - The space of bounded sequences with the mixed topology, Pacific J. Math. (to appear).

3. A. C. Cochran, Weak A-convex algebras, Proc. Amer. Math. Soc. 26 (1970), 73-77. MR 41 \#7435.

4. - Topological algebras and Mackey topology, Proc. Amer. Math. Soc. 30 (1971), 115-119. MR 45 \#897.

5. S. L. Gulick, The bidual of a locally multiplicatively convex algebra, Pacific J. Math. 17 (1966), 71-96. MR 34 \#627.

6. T. Ito and T. Seidman, Bounded generators of linear spaces, Pacific J. Math. 26 (1968), 283-287. MR 39 \#746.

7. G. Köthe, Topologische lineare Räume. I, Die Grundlehren der math. Wissenschaften, Band 107, Springer-Verlag, Berlin, 1960; 2nd ed., 1966; English transl., Die Grundlehren der math. Wissenschaften, Band 159, Springer-Verlag, New York, 1969. MR 24 \#A411; MR 33 \#3069; MR 40 \#1750.

8. S. Warner, Weak locally multiplicatively convex algebras, Pacific J. Math. 5 (1955), 1025-1032. MR 17, 876.

9. - Weakly topologized algebras, Proc. Amer. Math. Soc. 8 (1957), 314-316. MR 18, 911 .

Hindu College, University of Delhi, Delhi 7, India 